\title{
ON FUZZY FUNCTION SPACES
}

\section{GUNTHER JÄGER}

(Received 9 December 1996 and in revised form 20 October 1997)

\begin{abstract}
In [3], we started the investigation of compactness in fuzzy function spaces in FCS, the category of fuzzy convergence spaces as defined by Lowen/Lowen/Wuyts [8]. This paper goes somewhat deeper in the investigation of fuzzy function spaces using the notion of splitting and conjoining structures on fuzzy subsets. We discuss the connection to the exponential law and give several examples of such structures. As a special case, we study a notion of fuzzy compact open topology.
\end{abstract}

Keywords and phrases. Fuzzy topology, fuzzy convergence space, fuzzy function space, splitting structure, conjoining structure, pointwise convergence, continuous convergence, compact open topology.

1991 Mathematics Subject Classification. 54A40.

1. Introduction. The theory of fuzzy topological spaces is meanwhile highly developed. Especially, compactness and separation axioms have been thoroughly studied. Yet, one important field of classical topology has not yet attained wide attention in fuzzy topology: the theory of function spaces. Function spaces play an important role in functional analysis, in the theory of differential equations, in complex analysis, and in almost every other branch of modern mathematics, not to forget in topology itself. Therefore, it seems desirable to study function spaces also in fuzzy topology. In the meantime, three papers on this subject have appeared (Peng [11], Dang and Behera [2], and Alderton [1]). The fact that FTS, the category of fuzzy topological spaces (Lowen [9]), is not cartesian closed led Lowen and Lowen [7] to the definition of FCS, the category of fuzzy convergence spaces. This paper takes FCS as a starting point to discuss certain fuzzy function space structures via splitting and conjoining structures. It continues a previous paper by the author, where compactness in fuzzy function spaces in FCS was considered [3] and also considers function spaces in FTS.

2. Preliminaries. Let $X$ be a nonvoid set. Fuzzy subsets of $X$ are denoted by $A, B$, $C, \ldots \in[0,1]^{X}$, (ordinary) subsets of $X$ are denoted by small italics $a, b, c, \ldots \subset X$. For $a \subset X$, we denote by $\mathbf{1}_{a}$ the characteristic function of $a$ and in case $a=\{x\}$, we write $\mathbf{1}_{x}$. For the characteristic function of the whole set $X, Y, Z$, we write for short again $X, Y, Z$. The fundamental definitions of fuzzy set theory and fuzzy topology are assumed to be familiar to the reader. We especially take Lowen's definition of fuzzy topology [9]. In order to make this paper self-contained, however, we summarize the main results of our papers [3, 4,5]. Given a fuzzy subset $A \in[0,1]^{X}$, we denote $F_{X}(A):=\left\{B \in[0,1]^{X}: B \subset A\right\}$ and call $A_{0}:=\{x \in X: A(x)>0\}$ the support of $A$. For $A \in[0,1]^{X}$, we call a fuzzy subset of the form $B=A \cap \mathbf{1}_{B_{0}}$ a crisp fuzzy subset of $A$. 
If $A=X$, we regain the usual definition.

For a function $f: X \rightarrow Y$, we define its restriction on $A$ by $f \mid A(D):=f(D)(D \in$ $\left.F_{X}(A)\right)$ and the corresponding inverse image by $(f \mid A)^{-1}(E):=f^{-1}(E) \cap A\left(E \in F_{Y}(Y)\right)$ (cf. [4]). If, moreover, $f(A) \subset B$, we call $g=f \mid A: A \rightarrow B$ a fuzzy mapping from $A$ to $B$ [4].

A nonempty collection $\mathbb{F} \subset F_{X}(A)$ is called a fuzzy filter on $A$ if and only if it does not contain the empty fuzzy set $\varnothing:=\mathbf{1}_{\varnothing}$, is closed under finite intersections, and contains, for $F \in \mathbb{F}$, every fuzzy superset $A \supset G \supset F$. $\mathbb{B} \subset F_{X}(A)$ is called a fuzzy filter basis on $A$ if and only if it is not empty, does not contain the empty fuzzy set, and the intersection of two of its members contains a member of $\mathbb{B}$. For a fuzzy filter basis $\mathbb{B}$ on $A,[\mathbb{B}]_{A}=[\mathbb{B}]:=\{F \subset A: \exists B \in \mathbb{B}$ such that $B \subset F\}$ is a fuzzy filter on $A$. A fuzzy filter on $A$ is called a prime fuzzy filter if and only if whenever $F \cup G \in \mathbb{F}, F \in \mathbb{F}$ or $G \in \mathbb{F}$. For example, the fuzzy point filters $\left[\alpha \mathbf{1}_{x}\right]:=\{G \subset A: G(x) \geq \alpha\}(0<\alpha \leq A(x))$ are prime fuzzy filters. The set $\mathbb{F}(A)$ of fuzzy filters on $A$ is ordered by set inclusion. For $\mathbb{F} \in \mathbb{F}(A)$, the set $\mathbb{P}(\mathbb{F})$ of all prime fuzzy filters finer than $\mathbb{E}$ is inductive and, by Zorn's lemma, there exist minimal elements in $\mathbb{P}(\mathbb{F})$, the set of which is denoted by $\mathbb{P}_{m}(\mathbb{F})$ (cf. Lowen [10]). For a fuzzy filter $\mathbb{F} \in \mathbb{F}(A)$, the system $\imath(\mathbb{F}):=\left\{F_{0}: F \in \mathbb{F}\right\}$ is a filter on $A_{0}$. $\mathbb{F}$ is a prime fuzzy filter if and only if $\imath(\mathbb{F})$ is an ultrafilter. We further call for a fuzzy filter $\mathbb{F} \in \mathbb{F}(A)$

$$
c(\mathbb{F}):=\inf _{F \in \mathbb{F}} \sup _{x \in X} F(x)
$$

its characteristic value (Lowen/Lowen [6]).

For $A \in[0,1]^{X}$, we call a mapping

$$
\lim :\left\{\begin{array}{l}
\mathbb{F}(A) \longrightarrow F_{X}(A) \\
\mathbb{F} \longmapsto \lim \mathbb{F}
\end{array}\right.
$$

a fuzzy convergence on $A$ if and only if the following conditions are satisfied:

(PST) $\forall \mathbb{F} \in \mathbb{E}(A): \lim \mathbb{F}=\bigcap_{\mathbb{G} \in \mathbb{P} m(\mathbb{F})} \lim \mathbb{G}$;

(F1p) $\forall \mathbb{F} \in \mathbb{F}(A)$ prime fuzzy filters $: \lim \mathbb{F} \leq c(\mathbb{F})$;

(F2p) $\forall \mathbb{F}, \mathbb{G} \in \mathbb{F}(A)$ prime fuzzy filters : $\mathbb{F} \leq \mathbb{G} \Rightarrow \lim \mathbb{G} \subset \lim \mathbb{F}$;

(C1) $\forall x \in A_{0}, 0<\alpha \leq A(x): \alpha \mathbf{1}_{x} \subset \lim \left[\alpha \mathbf{1}_{x}\right]$.

(cf. $[5,7,8])$. The pair $(A, \lim )$ is then called a fuzzy convergence space (fcs for short).

A fuzzy topological space $(X, \Delta)$ can be considered as an fcs if we put, for $\mathbb{F} \in \mathbb{F}(X)$,

$$
\lim (\Delta) \mathbb{F}:=\bigcap_{\mathbb{G} \in \mathbb{P}_{m}(\mathbb{E})} \bigcap_{G \in \mathbb{G}} \bar{G}^{\Delta},
$$

which is the definition of limit of a fuzzy filter due to Lowen [10].

For two fuzzy convergences lim, lim' on the same fuzzy set $A \in[0,1]^{X}$, we say that $\lim ^{\prime}$ is finer than lim if and only if, for every prime fuzzy filter $\mathbb{F} \in \mathbb{F}(A)$, we have $\lim ^{\prime} \mathbb{F} \subset \lim \mathbb{E}$. We then write $\lim \leq \lim ^{\prime}$. It is easily verified that, for two fuzzy topologies $\Gamma, \Delta$ on $X$, we have $\Gamma \leq \Delta$ (i.e., $\Gamma \subset \Delta$ ) if and only if $\lim (\Gamma) \leq \lim (\Delta)$.

In [5], a fuzzy mapping $g:\left(A, \lim ^{A}\right) \rightarrow\left(B, \lim ^{B}\right)$ is called $\left(\lim ^{A}, \lim ^{B}\right)$-continuous (or simply continuous if the involved fuzzy convergences are clear) if and only if, for every prime fuzzy filter $\mathbb{F} \in \mathbb{F}(A)$, we have $g\left(\lim ^{A} \mathbb{F}\right) \subset \lim ^{B} g(\mathbb{F})$. By definition, we 
have, for two fuzzy convergences $\lim$, $\lim ^{\prime}$ on $A \in[0,1]^{X}$, lim $\leq \lim ^{\prime}$ if and only if $\operatorname{id}_{X} \mid A:\left(A, \lim ^{\prime}\right) \rightarrow(A, \lim )$ is continuous.

Now, let $\left(g_{\lambda}: A \rightarrow\left(A^{\lambda}, \lim _{\lambda}\right)\right)_{\lambda \in \Lambda}$ be a family of fuzzy mappings from a fuzzy set $A \in[0,1]^{X}$ to fcs's $\left(A^{\lambda}, \lim _{\lambda}\right)$ with $A^{\lambda} \in[0,1]^{X_{\lambda}}(\lambda \in \Lambda)$. If we put, for a prime fuzzy filter $\mathbb{F} \in \mathbb{F}(A)$,

$$
\operatorname{init}\left(\lim _{\lambda}, g_{\lambda}\right) \mathbb{F}:=\bigcap_{\lambda \in \Lambda} g^{-1}\left(\lim _{\lambda} g_{\lambda}(\mathbb{F})\right)
$$

and derive init $\left(\lim _{\lambda}, g_{\lambda}\right)$ for arbitrary fuzzy filters $\mathbb{F} \in \mathbb{F}(A)$ by (PST), then init $\left(\lim _{\lambda}, g_{\lambda}\right)$ is the coarsest fuzzy convergence on $A$ such that every $g_{\lambda}$ is continuous [5].

If $B \subset A\left(A\right.$, lim) fcs, and $\imath_{B}:=\operatorname{id}_{X} \mid B: B \rightarrow A$ is the fuzzy inclusion, we call $\left.\lim \right|_{B}:=$ init(lim, $\left.\imath_{B}\right)$ the fuzzy convergence on $B$ induced by $\lim$ and the pair $\left(B,\left.\lim \right|_{B}\right)$ a fuzzy subspace of $(A, \lim )$. We have $\left.\lim \right|_{B} \mathbb{F}=B \cap \lim [\mathbb{F}]$ for a prime fuzzy filter $\mathbb{F} \in \mathbb{F}(B)$. For more details on this subspace concept, see [3, 4, 5].

If $A^{\lambda} \in[0,1]^{X_{\lambda}}(\lambda \in \Lambda)$ and, as usual, $\Pi A^{\lambda}\left(\left(x_{\lambda}\right)\right):=\inf _{\lambda \in \Lambda} A^{\lambda}\left(x_{\lambda}\right)$ for $\left(x_{\lambda}\right) \in \Pi X_{\lambda}$, the restrictions $\pi_{\mu}:=\mathrm{pr}_{\mu} \mid \Pi A^{\lambda}$ of the projections $\mathrm{pr}_{\mu}: \Pi X_{\lambda} \rightarrow X_{\mu},\left(x_{\lambda}\right) \mapsto x_{\mu}$ are fuzzy mappings from $\Pi A^{\lambda}$ to $A^{\mu}(\mu \in \Lambda)$ (cf. [4]). If we denote $\pi-\lim :=\operatorname{init}\left(\lim _{\lambda}, \pi_{\lambda}\right)$, then $\left(\Pi A^{\lambda}, \pi-\lim \right)$ is called the product space of the fcs's $\left(\left(A^{\lambda}, \lim _{\lambda}\right)\right)_{\lambda \in \Lambda}$. For a prime fuzzy filter $\mathbb{F} \in \mathbb{F}\left(\Pi A^{\lambda}\right)$, we have $\pi-\lim \mathbb{F}=\Pi_{\lambda \in \Lambda} \lim _{\lambda} \pi_{\lambda}(\mathbb{F})=\bigcap_{\lambda \in \Lambda} \pi_{\lambda}^{-1}\left(\lim _{\lambda} \pi_{\lambda}(\mathbb{F})\right)$. For more details, we refer to [5].

If $g=f \mid A: A \rightarrow B$ and $h=k \mid C: C \rightarrow D$ are fuzzy mappings and if we define, as usual, the product-mapping $f \times k(a, c):=(f(a), k(c))$, then it is easily verified that $f \times k(A \times C) \subset B \times D$. Hence, we can define the fuzzy product-mapping $g \times h:=f \times k \mid$ $A \times C: A \times C \rightarrow B \times D$. The simple proofs of the next two propositions are left to the reader.

Proposition 2.1. Let, in the situation above, $F \subset A \times C$. If $\pi_{A}$ respectively $\pi_{C}$, are the fuzzy projections from $A \times C$ to $A$ respectively $C$, and $\pi_{B}$ respectively $\pi_{D}$, are the fuzzy projections from $B \times D$ to $B$ respectively $D$, then $g\left(\pi_{A}(F)\right)=\pi_{B}(g \times h(F))$ and $h\left(\pi_{C}(F)\right)=\pi_{D}(g \times h(F))$.

Proposition 2.2. Let $\left(A, \lim ^{A}\right),\left(B, \lim ^{B}\right),\left(C, \lim ^{C}\right)$, and $\left(D, \lim ^{D}\right)$ be fcs's and let $g: A \rightarrow B$ and $h: C \rightarrow D$ be continuous fuzzy mappings. Then the product-mapping $g \times h$ is $\left(\lim ^{A} \times \lim ^{C}, \lim ^{B} \times \lim ^{D}\right)$-continuous.

3. Continuous convergence on fuzzy subsets (The space $C(A, B)$ ). Let in this number $A \in[0,1]^{X}, B \in[0,1]^{Y}$, and always

$$
\left[\sup _{x \in X} A(X)\right] \mathbf{1}_{B_{0}} \subset B .
$$

We then have $B_{0}^{A_{0}}=\left\{g: A_{0} \rightarrow B_{0} \mid g(A) \subset B\right\}$, i.e., we do not need to distinguish between mappings from $A_{0}$ to $B_{0}$ and fuzzy mappings from $A$ to $B$. For a mapping $g: A_{0} \rightarrow B_{0}$, we put

$$
B^{A}(g)=\eta(g):=\inf _{x \in A_{0}} B(g(x))
$$


i.e., $B^{A}=\Pi_{x \in A_{0}} B$ (identifying $g$ and $(g(x))_{x \in A_{0}}$ in the natural way) is a fuzzy set on $B_{0}^{A_{0}}$.

We consider the evaluation map

$$
\mathrm{ev}:\left\{\begin{array}{l}
B_{0}^{A_{0}} \times A_{0} \longrightarrow B_{0} \\
(g, x) \longmapsto g(x) .
\end{array}\right.
$$

If (3.1) is assumed, then ev : $B^{A} \times A \rightarrow B$ is a fuzzy mapping [3]. We further denote by $\pi_{B^{A}}: B^{A} \times A \rightarrow B^{A}$ the restriction of the projection $\operatorname{pr}_{B_{0}^{A_{0}}}:(\mathfrak{g}, x) \mapsto g$ and by $\pi_{A}$ : $B^{A} \times A \rightarrow A$ the restriction of the projection $\operatorname{pr}_{A_{0}}:(g, x) \mapsto x$ on $B^{A} \times A$. The fuzzy convergence structure of continuous convergence on $B^{A}$ is defined as follows (cf. [3, $7,8])$. For $\left(A, \lim ^{A}\right),\left(B, \lim ^{B}\right)$ fcs's, $g: A \rightarrow B$ a fuzzy mapping and $\mathbb{F} \in \mathbb{F}\left(B^{A}\right)$ a prime fuzzy filter, we put

$$
\begin{aligned}
C(\mathbb{F}, g):=\{ & \left\{\alpha \in[0,1] \mid \forall \Theta \in \mathbb{F}\left(B^{A} \times A\right)\right. \text { prime fuzzy filters such that } \\
& \left.\pi_{B^{A}}(\Theta) \leq \mathbb{E}, \forall x \in A_{0}: \lim ^{A} \pi_{A}(\Theta)(x) \wedge \alpha \leq \pi_{x}^{-1}\left(\lim ^{B} \operatorname{ev}(\Theta)\right)(g)\right\}
\end{aligned}
$$

and

$$
\mathrm{c}-\lim \mathbb{F}(g):=c(\mathbb{F}) \wedge \eta(g) \wedge \sup C(\mathbb{F}, g) .
$$

For an arbitrary fuzzy filter $\mathbb{E} \in \mathbb{F}\left(B^{A}\right)$, we derive c-lim $\mathbb{F}$ by (PST). c-lim then satisfies (PST), (F1p), and (F2p) and, in general, fails to satisfy (C1). Therefore, we speak of c-lim as a "weak fuzzy convergence structure" (cf. [3]).

If $M \subset B^{A}$ is a fuzzy subset of $B^{A}$, we call c-lim $\left.\right|_{M}$ the (weak) fuzzy convergence structure of continuous convergence on $M$ and denote this (weak) fuzzy convergence again by c-lim. The next proposition shows that we can calculate the fuzzy convergence of continuous convergence for certain $M \subset B^{A}$ "from inside."

Proposition 3.1. Let $\left(A, \lim ^{A}\right),\left(B, \lim ^{B}\right)$ be fcs's and $M=B^{A} \cap \mathbf{1}_{M_{0}}$ be a crisp fuzzy subset of $B^{A}$. If we put for a prime fuzzy filter $\mathbb{F} \in \mathbb{F}(M)$ and $g \in M_{0}$

$$
\begin{aligned}
C_{M}(\mathbb{F}, g):= & \{\alpha \in[0,1] \mid \forall \Theta \in \mathbb{F}(M \times A) \text { prime fuzzy filter such that } \\
& \left.\pi_{M}(\Theta) \leq \mathbb{F}, \forall x \in A_{0}: \lim ^{A} \pi_{A}(\Theta)(x) \wedge \alpha \leq \pi_{x}^{-1}\left(\lim ^{B} \operatorname{ev}(\Theta)\right)(g)\right\},
\end{aligned}
$$

then $c-\lim \mathbb{F}(g)=c(\mathbb{F}) \wedge \eta(g) \wedge \sup C_{M}(\mathbb{F}, g)$. Here, $\pi_{M}=\operatorname{pr}_{M_{0}} \mid M \times A$ is the restriction of the mapping $M_{0} \times A_{0} \ni(g, x) \mapsto g \in M_{0}$ and $\pi_{A}: M \times A \rightarrow A$ is the fuzzy projection and $\mathrm{ev}: M \times A \rightarrow B$ and $\pi_{x}=\operatorname{pr}_{x} \mid M: M \rightarrow B$ is the restriction of the mapping $g \mapsto g(x)$.

Proof. We prove that $C([\mathbb{F}], g)=C_{M}(\mathbb{F}, g)$. Let $\alpha \in C([\mathbb{F}], g)$ and let $\Theta \in \mathbb{F}(M \times A)$ be a prime fuzzy filter such that $\pi_{M}(\Theta) \leq \mathbb{F}$ and let $x \in A_{0}$. Then $[\Theta] \in \mathbb{F}\left(B^{A} \times A\right)$ is a prime fuzzy filter and $\left[\pi_{M}(\Theta)\right] \leq[\mathbb{F}]$. For $\Psi \subset M \times A$ and $g \in M_{0}$, we have $\pi_{M}(\Psi)(g)=$ $\sup _{x \in A_{0}} \Psi(g, x)=\pi_{B^{A}}(\Psi)(g)$ which yields $\left[\pi_{M}(\Theta)\right]=\pi_{B^{A}}([\Theta])$. Obviously, $\pi_{A}([\Theta])=$ $\pi_{A}(\Theta)$ and $\operatorname{ev}([\Theta])=\operatorname{ev}(\Theta)$ (here, the fuzzy functions on the left sides are defined on $B^{A} \times A$ and the ones on the right sides are defined on $\left.M \times A\right)$. Hence, we get

$$
\begin{aligned}
\lim ^{A} \pi_{A}(\Theta)(x) \wedge \alpha & =\lim ^{A} \pi_{A}([\Theta])(x) \wedge \alpha \\
& \leq \pi_{x}^{-1}\left(\lim ^{B} \operatorname{ev}([\Theta])\right)(g) \\
& =\pi_{x}^{-1}\left(\lim ^{B} \operatorname{ev}(\Theta)\right)(g),
\end{aligned}
$$

i.e., $\alpha \in C_{M}(\mathbb{F}, g)$. 
Conversely, let $\alpha \in C_{M}(\mathbb{F}, g)$ and $\Theta \in \mathbb{F}\left(B^{A} \times A\right)$ be a prime fuzzy filter such that $\pi_{B^{A}}(\Theta) \leq[\mathbb{F}]$ and let $x \in A_{0}$. Then, as $\imath\left(\pi_{B^{A}}(\Theta)\right)=\imath([\mathbb{F}])$ and $M$ is a crisp fuzzy subset, we get that $M \in \pi_{B^{A}}(\Theta)$ and, therefore, also $M \times A \in \Theta$. We put $\Theta^{\prime}:=\Theta_{M \times A}=$ $\{\theta \cap(M \times A): \theta \in \Theta\}$. Then $\left[\Theta^{\prime}\right]=\Theta$, and $\Theta^{\prime}$ is also a prime fuzzy filter. A simple computation shows that $\pi_{M}(\Psi \cap(M \times A))=\pi_{B^{A}}(\Psi) \cap M$ for $\Psi \in B^{A} \times A$ and so $\pi_{M}\left(\Theta^{\prime}\right)=\left(\pi_{B^{A}}(\Theta)\right)_{M}$. From this, we conclude that $\pi_{M}\left(\Theta^{\prime}\right) \leq([\mathbb{F}])_{M}=\mathbb{F}$. As, furthermore, $\pi_{A}\left(\Theta^{\prime}\right)=\pi_{A}(\Theta)$ and $\operatorname{ev}\left(\Theta^{\prime}\right)=\operatorname{ev}(\Theta)$ (where again the fuzzy functions on the left sides are defined on $M \times A$ and those on the right sides are defined on $B^{A} \times A$ ), this yields $\alpha \in C([\mathbb{F}], g)$ which completes the proof.

Proposition 3.2. Let $\left(A, \lim ^{A}\right),\left(B, \lim ^{B}\right)$ be fcs's and $M=B^{A} \cap \mathbf{1}_{M_{0}}$ be a crisp fuzzy subset of $B^{A}$. The following hold:

(i) ev: $\left(M \times A, c\right.$-lim $\left.\times \lim ^{A}\right) \rightarrow\left(B, \lim ^{B}\right)$ is continuous.

(ii) If $A=\alpha \mathbf{1}_{X}, B=\alpha \mathbf{1}_{Y}$ and lim* is a fuzzy convergence on $M$ such that $\mathrm{ev}:(M \times$ $\left.A, \lim ^{*} \times \lim ^{A}\right) \rightarrow\left(B, \lim ^{B}\right)$ is continuous, then $c$-lim $\leq \lim *$.

Proof. Using Proposition 3.1, we can copy the corresponding proof of [3, Prop. 4.6].

We now put for two fcs's $\left(A, \lim ^{A}\right)$ and $\left(B, \lim ^{B}\right)$

$$
C(A, B)_{0}:=\left\{g:\left(A, \lim ^{A}\right) \longrightarrow\left(B, \lim ^{B}\right) \text { continuous }\right\}
$$

and define the fuzzy subset $C(A, B)$ of $B^{A}$ by $C(A, B):=B^{A} \cap \mathbf{1}_{C(A, B)_{0}}$. In [3, Prop. 4.2], we showed that, for a continuous fuzzy mapping $g:\left(A, \lim ^{A}\right) \rightarrow\left(B, \lim ^{B}\right)$ and for $0<\alpha \leq \eta(g)$, we have $\alpha \mathbf{1}_{g} \subset \mathrm{c}-\lim \left[\alpha \mathbf{1}_{g}\right]$. Hence, $(C(A, B), \mathrm{c}-\lim )$ satisfies the axiom (C1), i.e., is an fcs.

We finally mention a result due to Lowen/Lowen [7]. Let $X, Y, Z$ be nonvoid sets and $f: X \times Y \rightarrow Z$ be a mapping. We define a mapping $\varphi(f): X \rightarrow Z^{Y}, \varphi(f)(x):=f(x, \cdot)$. The just-defined bijection $\varphi: Z^{X \times Y} \rightarrow\left(Z^{Y}\right)^{X}$ is called an "exponential map" (Poppe [12]).

Proposition 3.3. Let $\left(X, \lim ^{X}\right),\left(Y, \lim ^{Y}\right)$ and $\left(Z, \lim ^{Z}\right)$ be $f c s$ 's. Then

$$
\varphi(C(X \times Y, Z))=C(X, C(Y, Z)) .
$$

Here, $C(Y, Z)$ is provided with the fuzzy convergence of continuous convergence and $X \times Y$ with the product fuzzy convergence $\lim ^{X} \times \lim ^{Y}$.

Proof. $\varphi(C(X \times Y, Z)) \subset C(X, C(Y, Z))$ is shown in [7, Thm. 5.2]. The reverse inclusion follows using the continuity of the evaluation map (Proposition 3.2) and the continuity of the fuzzy product-mapping (Proposition 2.2) in exactly the same way as the proof of the corresponding "classical" theorem 2.2, (a) $\Rightarrow(b)$ in Poppe [12].

\section{Splitting and conjoining fuzzy convergences}

Definition 4.1. Let $\left(A, \lim ^{A}\right),\left(B, \lim ^{B}\right)$ be fcs's, $M \subset B^{A}$ and $\lim$ be a fuzzy convergence on $M$.

(i) $\lim$ is called conjoining for $M$ if and only if c-lim $\leq \lim$ holds on $M$. 
(ii) $\lim$ is called splitting for $M$ if and only if $\lim \leq \mathrm{c}$-lim holds on $M$.

As an immediate consequence of Proposition 3.2, we obtain the following proposition.

Proposition 4.2. Let $\left(A, \lim ^{A}\right),\left(B, \lim ^{B}\right)$ be $f c s$ 's and $M \subset B^{A}$. The following hold:

(i) If $\lim$ is conjoining for $M$, then $\mathrm{ev}:\left(M \times A, \lim \times \lim ^{A}\right) \rightarrow\left(B, \lim ^{B}\right)$ is continuous.

(ii) If $A=\alpha \mathbf{1}_{X}, B=\alpha \mathbf{1}_{Y}$ and $M=B^{A} \cap \mathbf{1}_{M_{0}}$ is a crisp fuzzy subset, then, from the continuity of $\mathrm{ev}:\left(M \times A, \lim \times \lim ^{A}\right) \rightarrow\left(B, \lim ^{B}\right)$, we get that $\lim$ is conjoining.

Splitting and conjoining fuzzy convergences are closely related to the "exponential law" (for corresponding "classical” results (cf. Poppe [12])).

Proposition 4.3. Let $\left(Y, \lim ^{Y}\right),\left(Z, \lim ^{Z}\right)$ be fcs's and $\lim$ be a fuzzy convergence for $C(Y, Z)$. Then the following are equivalent:

(i) lim is splitting for $C(Y, Z)$,

(ii) for each $f c s\left(X, \lim ^{X}\right)$, we have $\varphi(C(X \times Y, Z)) \subset C(X,(C(Y, Z)$, lim)).

Proof. Let first lim be splitting for $C(Y, Z)$. From Proposition 3.3, we get that, for a continuous fuzzy mapping $f: X \times Y \rightarrow Z$, the fuzzy mapping $\varphi(f): X \rightarrow$ $(C(Y, Z), \mathrm{c}-\lim )$ is continuous. Hence, for a prime fuzzy filter $\mathbb{F} \in \mathbb{F}(X)$, we have

$$
\varphi(f)\left(\lim ^{X} \mathbb{F}\right) \subset c-\lim \varphi(f)(\mathbb{F}) \subset \lim \varphi(f)(\mathbb{F}),
$$

i.e., $\varphi(f)$ is $\left(\lim ^{X}, \lim \right)$-continuous.

Conversely, let condition (ii) hold. ( $C(Y, Z)$, c-lim) is a fuzzy convergence space and, hence,

$$
\varphi(C((C(Y, Z), \mathrm{c}-\lim ) \times Y, Z)) \subset C((C(Y, Z), \mathrm{c}-\lim ),(C(Y, Z), \lim )) .
$$

By Proposition 3.2, the evaluation map ev is continuous, i.e., ev $\in C((C(Y, Z), \mathrm{c}-\lim ) \times$ $Y, Z)$ and, hence, $\varphi(\mathrm{ev}) \in C((C(Y, Z), \mathrm{c}-\lim ),(C(Y, Z), \lim ))$. As $\varphi(\mathrm{ev})(g)(y)=$ $\operatorname{ev}(g, \cdot)(y)=\operatorname{ev}(g, y)=g(y)=\operatorname{id}_{C(Y, Z)}(g)(y)$, i.e., $\varphi(\mathrm{ev})$ is the identity map on $C(Y, Z)$, we conclude that $\lim \leq$ c-lim, i.e., $\lim$ is splitting for $C(Y, Z)$.

Proposition 4.4. Let $\left(Y, \lim ^{Y}\right),\left(Z, \lim ^{Z}\right)$ be fcs's and $\lim$ be a fuzzy convergence for $C(Y, Z)$. Then the following are equivalent:

(i) $\lim$ is conjoining for $C(Y, Z)$,

(ii) for each $f c s\left(X, \lim ^{X}\right)$, we have $C(X,(C(Y, Z), \lim )) \subset \varphi(C(X \times Y, Z))$.

Proof. Let first lim be conjoining for $C(Y, Z)$, and let $f:\left(X, \lim ^{X}\right) \rightarrow(C(Y, Z), \lim )$ be continuous. As c-lim $\leq \lim$, then also $f:\left(X, \lim ^{X}\right) \rightarrow(C(Y, Z), \mathrm{c}-\lim )$ is continuous. This yields $C(X,(C(Y, Z), \lim )) \subset C(X,(C(Y, Z), \mathrm{c}-\lim ))$. Proposition 3.3 now implies condition (ii).

Conversely, let condition (ii) hold. As $(C(Y, Z), \lim )$ is an fcs, we obtain

$$
C((C(Y, Z), \lim ),(C(Y, Z), \lim )) \subset \varphi((C(Y, Z), \lim ) \times Y, Z) .
$$

As $\operatorname{id}_{C(Y, Z)}$ is (lim, lim)-continuous, we deduce, herefrom, that

$$
\varphi^{-1}\left(\operatorname{id}_{C(Y, Z)}\right):(C(Y, Z), \lim ) \times Y \rightarrow Z
$$


is continuous. But, as $\varphi(\mathrm{ev})=\operatorname{id}_{C(Y, Z)}$ and $\varphi$ is a bijection, we get $\varphi^{-1}\left(\operatorname{id}_{C(Y, Z)}\right)=\mathrm{ev}$ and, hence, by Proposition 4.2, lim is conjoining for $C(Y, Z)$.

\section{Examples for splitting and conjoining fuzzy convergences}

5.1. The discrete and the indiscrete fuzzy convergences. Let $A \in[0,1]^{X}$. If we put for a prime fuzzy filter $\mathbb{F} \in \mathbb{F}(A)$

$$
\begin{gathered}
\qquad \lim _{\imath} \mathbb{F}:=c(\mathbb{F}) \mathbf{1}_{X} \cap A, \\
\lim _{\delta} \mathbb{F}(x):=\left\{\begin{array}{ll}
c(\mathbb{F}) & \text { if and only if } \imath(\mathbb{F})=[x], \\
0 & \text { else }
\end{array} \quad(x \in X)\right.
\end{gathered}
$$

and derive $\lim _{\imath}$ respectively $\lim _{\delta}$ for arbitrary fuzzy filters on $A$ by (PST), then the following proposition holds.

Proposition 5.1. Let $A \in[0,1]^{X}$. Then

(i) $\lim _{i}, \lim _{\delta}$ are fuzzy convergences on $A$,

(ii) for a fuzzy convergence $\lim$ on $A$, we have $\lim _{\iota} \leq \lim \leq \lim _{\delta}$.

Proof. (i) That $\lim _{\imath}$ is a fuzzy convergence on $A$ is obvious and, obviously, $\lim _{\delta}$ satisfies axioms (F1p), (PST), and (C1). If $\mathbb{F}, \mathbb{G} \in \mathbb{F}(A)$ are prime fuzzy filters and $\mathbb{F} \leq \mathbb{G}$ and $\lim _{\delta} \mathbb{G}(x)>0$, then $\imath(\mathbb{G})=[x]$ and, consequently, also $\imath(\mathbb{F})=[x]$. Hence, $\lim _{\delta} \mathbb{F}(x)=c(\mathbb{F}) \geq c(\mathbb{G})=\lim _{\delta} \mathbb{G}(x)$ and also (F2p) holds.

(ii) Let $\mathbb{F} \in \mathbb{F}(A)$ be a prime fuzzy filter. As an immediate consequence of (F1p), we obtain $\lim \mathbb{F} \subset \lim _{\imath} \mathbb{F}$. Now, let $\lim _{\delta} \mathbb{F}(x)=c(\mathbb{F})>0$. Then $\imath(\mathbb{F})=[x]$ and, hence, $A \cap \mathbf{1}_{x} \in \mathbb{F}$. From this, we conclude that, for $F \in \mathbb{F}$,

$$
F(x)=F \cap\left(A \cap \mathbf{1}_{x}\right)(x)=\sup _{y \in X} F \cap A \cap \mathbf{1}_{x}(y) \geq \inf _{G \in \mathbb{F}} \sup _{y \in X} G(x)=c(\mathbb{F}),
$$

i.e., $\mathbb{F} \leq\left[c(\mathbb{F}) \mathbf{1}_{x}\right]$. Hence, it follows, by $(\mathrm{F} 2 \mathrm{p})$ and $(\mathrm{C} 1)$, that

$$
\lim \mathbb{F}(x) \geq \lim \left[c(\mathbb{F}) \mathbf{1}_{x}\right](x) \geq c(\mathbb{F}) \mathbf{1}_{x}(x)=c(\mathbb{F}),
$$

i.e., $\lim \mathbb{F} \supset \lim _{\delta} \mathbb{F}$ and the proposition is proved.

$\lim _{\imath}$ is called the indiscrete fuzzy convergence on $A$ and $\lim _{\delta}$ is called the discrete fuzzy convergence on $A$.

Corollary 5.2. Let $\left(A, \lim ^{A}\right),\left(B, \lim ^{B}\right)$ be $f c s$ 's and $M \subset B^{A}$. Then

(i) The indiscrete fuzzy convergence on $M$ is splitting for $M$.

(ii) If $M \subset C(A, B)$, then the discrete fuzzy convergence on $M$ is conjoining for $M$.

5.2. The fuzzy convergence of pointwise convergence. Let $\left(A, \lim ^{A}\right),\left(B, \lim ^{B}\right)$ be fcs's. We define a fuzzy convergence on $B^{A}$ by putting, for a prime fuzzy filter $\mathbb{F} \in$ $\mathbb{F}\left(B^{A}\right)$,

$$
p-\lim \mathbb{F}:=\bigcap_{x \in A_{0}} \pi_{x}^{-1}\left(\lim ^{B} \pi_{x}(\mathbb{F})\right)
$$

and derive $p$-lim $\mathbb{E}$ for an arbitrary fuzzy filter on $B^{A}$ by (PST), i.e., $p$-lim is the initial fuzzy convergence on $B^{A}$ respectively the $\left(\pi_{x}, B\right)_{x \in A_{0}} . p$-lim is called the fuzzy 
convergence of pointwise convergence on $B^{A}$ [3]. In [3], we proved the following proposition.

Proposition 5.3. Let $A \in[0,1]^{X}, B \in[0,1]^{Y}$ be constant fuzzy sets of the same height $\alpha>0$ and $\left(A, \lim ^{A}\right),\left(B, \lim ^{B}\right)$ be fcs's. Then $\mathrm{p}-\lim \leq c$-lim.

COROLLARY 5.4. Under the assumptions of Proposition 5.3, the fuzzy convergence of pointwise convergence $\mathrm{p}$-lim is splitting for $B^{A}$.

5.3. The fuzzy convergence of strictly continuous convergence. Let $\left(A, \lim ^{A}\right)$, $\left(B, \lim ^{B}\right)$ be fcs's and $M \subset B^{A}$. We put for a prime fuzzy filter $\mathbb{F} \in \mathbb{F}(M)$ and a fuzzy mapping $g: A \rightarrow B$

$$
\begin{aligned}
& \operatorname{SC}_{M}(\mathbb{F}, g):=\{\alpha \in[0,1] \mid \forall \Theta \in \mathbb{F}(M \times A) \text { prime fuzzy filters such that } \\
&\left.\pi_{M}(\Theta) \leq \mathbb{F}, \forall y \in B_{0}: \lim ^{B} \mathcal{g}\left(\pi_{A}(\Theta)\right)(y) \wedge \alpha \leq \lim ^{B} \operatorname{ev}(\Theta)(y)\right\},
\end{aligned}
$$

and define

$$
\operatorname{sc}-\lim \mathbb{F}(g):=c(\mathbb{F}) \wedge \eta(g) \wedge \operatorname{supSC}_{M}(\mathbb{F}, g),
$$

and derive $s c$ - $\lim \mathbb{E}$ for arbitrary fuzzy filters on $M$ by (PST).

Proposition 5.5. Under the assumptions mentioned above, sc-lim is a fuzzy convergence on $M$.

Proof. By definition, sc-lim satisfies (PST) and (F1p). If $\mathbb{F} \leq \mathbb{G}$ for two prime fuzzy filters $\mathbb{F}, \mathbb{G} \in \mathbb{F}(M)$, we obviously have $\mathrm{SC}_{M}(\mathbb{G}, g) \subset \mathrm{SC}_{M}(\mathbb{F}, g)$ and, hence, sc-lim $\mathbb{G} \subset$ $\mathrm{sc}-\lim \mathbb{F}$, i.e., (F2p) holds. So, all that remains to be shown is (C1): Let $0<\alpha \leq \eta(g)$ and $\Theta \in \mathbb{F}(M \times A)$ be a prime fuzzy filter such that $\pi_{M}(\Theta) \leq\left[\alpha \mathbf{1}_{g}\right]$. It then follows, in exactly the same way as in the proof of [7, Prop. 5.1], that $\operatorname{ev}(\Theta) \leq g\left(\pi_{A}(\Theta)\right)$. Using (F2p) for $\left(B, \lim ^{B}\right)$, we deduce herefrom $\lim ^{B} g\left(\pi_{A}(\Theta)\right) \subset \lim ^{B} \mathrm{ev}(\Theta)$. Hence, $1 \in \mathrm{SC}_{M}\left(\left[\alpha \mathbf{1}_{g}\right], g\right)$ and, therefore, sc- $\lim \left[\alpha \mathbf{1}_{g}\right](g)=c\left(\left[\alpha \mathbf{1}_{g}\right]\right)=\alpha$ and sc-lim satisfies (C1). (sc-lim is called the fuzzy convergence of strictly continuous convergence on $M$.)

Proposition 5.6. If $A=\alpha \mathbf{1}_{X}$ and $B=\alpha \mathbf{1}_{Y}$, then sc -lim is conjoining for $C(A, B)$.

Proof. We put $M:=C(A, B)$. Let $\mathbb{F} \in \mathbb{F}(M)$ be a prime fuzzy filter and $g \in C(A, B)_{0}$. Further, let $\alpha \in \mathrm{SC}_{M}(\mathbb{F}, g)$ and $\Theta \in \mathbb{F}(M \times A)$ be a prime fuzzy filter such that $\pi_{M}(\Theta) \leq \mathbb{F}$ and let $x \in A_{0}$. Then $g(x) \in B_{0}$ and the continuity of $g$ yields

$$
\begin{aligned}
\lim ^{A} \pi_{A}(\Theta)(x) \wedge \alpha & \leq g\left(\lim ^{A} \pi_{A}(\Theta)\right)(g(x)) \wedge \alpha \\
& \leq \lim ^{B} g\left(\pi_{A}(\Theta)\right)(g(x)) \wedge \alpha \\
& \leq \lim ^{B} \operatorname{ev}(\Theta)(g(x)) \\
& =\pi_{x}^{-1}\left(\lim ^{B} \operatorname{ev}(\Theta)\right)(g) .
\end{aligned}
$$

Hence, $\alpha \in C_{M}(\mathbb{F}, g)$ and so sc- $\lim \geq \mathrm{c}-\lim$ on $M$. 
6. Splitting and conjoining fuzzy topologies. In this number, we only consider fuzzy topological spaces $(X, \Delta),(Y, \Gamma)$ in the sense of Lowen [9] and crisp subspaces of $Y^{X}$. As in the (classical) theory of convergence space (Poppe [12]), we call a fuzzy topology $\mathbf{T}$ on a crisp subset $M=\mathbf{1}_{M_{0}} \subset Y^{X}$ splitting (resp. conjoining) for $M$ if and only if the corresponding fuzzy convergence $\lim (\mathbf{T})$ is splitting (resp. conjoining) for $M$.

6.1. The discrete and the indiscrete fuzzy topologies. Let $X$ be a nonvoid set. If we put $\Delta_{\delta}:=F_{X}(X)$ and $\Delta_{\imath}:=\{\underline{\alpha}: 0 \leq \alpha \leq 1\}$, then, obviously, $\Delta_{\delta}$ and $\Delta_{\imath}$ are fuzzy topologies on $X . \Delta_{\delta}$ is called discrete fuzzy topology on $X$ and $\Delta_{l}$ is called the indiscrete fuzzy topology on $X$.

Proposition 6.1. We have $\lim \left(\Delta_{\delta}\right)=\lim _{\delta}$ and $\lim \left(\Delta_{l}\right)=\lim _{\imath}$.

Proof. First, we show that $\lim \left(\Delta_{\delta}\right)=\lim _{\delta}$. Let $\mathbb{F} \in \mathbb{F}(X)$ be a prime fuzzy filter. By the definition of $\lim \left(\Delta_{\delta}\right)$, We obtain $\lim \left(\Delta_{\delta}\right) \mathbb{F}=\bigcap_{F \in \mathbb{F}} F$. If $\lim \left(\Delta_{\delta}\right) \mathbb{F}=\varnothing$, assume that $\lim _{\delta} \mathbb{F}(x)>0$. Then $\imath(\mathbb{F})=[x]$ and, therefore, $\mathbb{F} \leq\left[c(\mathbb{F}) \mathbf{1}_{x}\right]$ (see the proof of Proposition 5.1). (F2p) together with (C1) for $\lim \left(\Delta_{\delta}\right)$ then imply $\varnothing=\lim \left(\Delta_{\delta}\right) \mathbb{F} \supset$ $c(\mathbb{F}) \mathbf{1}_{x}$, a contradiction to $\lim _{\delta} \mathbb{F}(x)>0$. Hence, also $\lim _{\delta} \mathbb{F}=\varnothing \cdot \operatorname{If} \lim \left(\Delta_{\delta}\right) \mathbb{F}(x)=: \alpha>$ $0<\beta:=\lim \left(\Delta_{\delta}\right) \mathbb{F}(y)$, then $\mathbb{F} \leq\left[\alpha \mathbf{1}_{x}\right]$ and $\mathbb{F} \leq\left[\beta \mathbf{1}_{y}\right]$. Thus, $\imath(\mathbb{F})=[x]$ and $\imath(\mathbb{F})=[y]$, i.e., $x=y$. From this, we conclude that, $\lim \left(\Delta_{\delta}\right) \mathbb{F}=\alpha \mathbf{1}_{x} \leq c(\mathbb{F}) \mathbf{1}_{x}=\lim _{\delta} \mathbb{F}$. But as $\imath(\mathbb{F})=[x]$, we again get $\mathbb{F} \leq\left[c(\mathbb{F}) \mathbf{1}_{x}\right]$ and this yields, using $(\mathrm{F} 2 \mathrm{p}), \lim \left(\Delta_{\delta}\right) \mathbb{F} \supset c(\mathbb{F}) \mathbf{1}_{x}$. Hence, $\lim \left(\Delta_{\delta}\right)=\lim _{\delta}$ is established.

To prove $\lim \left(\Delta_{\imath}\right)=\lim _{\imath}$ it suffices to remark that, for $F \in[0,1]^{X}$, we have $\bar{F}^{\Delta_{\imath}}=$ $\sup _{x \in X} F(x)$ and so $\lim \left(\Delta_{\imath}\right) \mathbb{F}=\inf _{F \in \mathbb{F}} \sup _{x \in X} F(x)=c(\mathbb{F})$ for a prime fuzzy filter $\mathbb{F} \in \mathbb{F}(X)$.

COROLLARY 6.2. Let $(X, \Delta)$ and $(Y, \Gamma)$ be fuzzy topological spaces and $M=\mathbf{1}_{M_{0}} \subset Y^{X}$ be a crisp fuzzy subset of $Y^{X}$. Then

(i) The indiscrete fuzzy topology on $M$ is splitting for $M$.

(ii) If $M \subset C(X, Y)$, then the discrete fuzzy topology is conjoining for $M$.

6.2. The fuzzy compact open topology. In [11], Peng defines a notion of fuzzy compact open topology using the notion of $N$-compactness due to Wang [13]. Here, we alter his definition slightly using Lowen's [9] definition of compactness. Let $(X, \Delta)$, $(Y, \Gamma)$ be fuzzy topological spaces. For a subset $k \subset X$ and $G \in[0,1]^{Y}$, we put

$$
\left(\mathbf{1}_{k}, G\right)(g):=\inf _{x \in k} G(g(x)) .
$$

So, $\left(\mathbf{1}_{k}, G\right) \in[0,1]^{Y}$. It is easily verified that the system

$$
\left\{\left(\mathbf{1}_{k}, G\right): \mathbf{1}_{k} \text { compact in }(X, \Delta), G \in \Gamma\right\}
$$

is a subbase for a fuzzy topology on $Y^{X}$, the fuzzy compact open topology $\Delta_{\text {co }}$ on $Y^{X}$. If we restrict the functions $\left(\mathbf{1}_{k}, G\right)$ on $C(X, Y)$, then the subspace topology of $\Delta_{\text {co }}$ is also denoted by $\Delta_{\text {co }}$.

For the proof of the next proposition, we need two lemmas, the proofs of which are left to the reader. 
LEMMA 1. Let $X$ be a nonvoid set and $\Delta, \Gamma$ be fuzzy topologies on $X$. If, for every $D \in \Gamma$ and for every $x \in X$ such that $D(x)=: \alpha_{x}>0$, there is $H_{x} \in \Delta$ such that $H_{x} \cap \underline{\alpha_{x}} \subset D$ and $H_{x}(x) \wedge \alpha_{x}=D(x)$, then $\Delta \geq \Gamma$.

LEMMA 2. Let $(X, \Delta)$ and $(Y, \Gamma)$ be fuzzy topological spaces and let $(X \times Y, \Delta \times \Gamma)$ be their product space. Then $G \in \Delta \times \Gamma$ if and only if, for every $(x, y) \in X \times Y$ and every $\epsilon>0$, there are fuzzy sets $H_{x}^{\epsilon} \in \Delta, K_{y}^{\epsilon} \in \Gamma$ such that $H_{x}^{\epsilon} \times K_{y}^{\epsilon} \subset G$ and $H_{x}^{\epsilon} \times K_{y}^{\epsilon}(x, y) \geq$ $G(x, y)-\epsilon$.

Proposition 6.3. Let $(X, \Delta)$ and $(Y, \Gamma)$ be fuzzy topological spaces and $\mathrm{T}$ be a conjoining fuzzy topology on $Y^{X}$ (respectively $C(X, Y)$ ). Then $\mathbf{T} \geq \Delta_{\text {co }}$.

Proof. Let $\mathbf{1}_{k} \in[0,1]^{X}$ be compact and $G \in \Gamma$ and $\left(\mathbf{1}_{k}, G\right)(g)=\inf _{x \in k} G(g(x))=$ : $\alpha_{g}>0$. Then, for every $x \in k$, we have $\alpha_{g} \leq G(g(x))=\mathrm{ev}^{-1}(G)(g, x)$. As T is conjoining, the evaluation map is continuous (Proposition 4.2 and [5, Prop. 4.3]) and, hence, $\mathrm{ev}^{-1}(G) \in \mathbf{T} \times \Delta$. Let $\epsilon>0$. Lemma 2 yields the existence of fuzzy sets $H_{x} \in \mathbf{T}, O_{x} \in \Delta$ such that

$$
\begin{gathered}
H_{x} \times O_{x} \subset \mathrm{ev}^{-1}(G), \\
H_{x}(g) \wedge O_{x}(x) \geq \mathrm{ev}^{-1}(G)(g, x)-\frac{\epsilon}{2} \geq \alpha_{g}-\frac{\epsilon}{2} .
\end{gathered}
$$

From this, we see that the system $\left\{O_{x}: x \in k\right\}$ is an open cover of $\left(\alpha_{g}-(\epsilon / 2)\right) \mathbf{1}_{k}$. As $\mathbf{1}_{k}$ is compact, there are finitely many $x_{\epsilon}^{1}, \ldots, x_{\epsilon}^{n} \in k$ such that $\bigcup_{k=1}^{n} O_{x_{\epsilon}^{k}} \supset\left(\alpha_{g}-\epsilon\right) \mathbf{1}_{k}$. We put $H:=\bigcap_{k=1}^{n} H_{x_{\epsilon}^{k}}$. Then $H \in \mathbf{T}$. We prove that $H \cap \alpha_{g} \subset\left(\mathbf{1}_{k}, G\right)$ in two steps.

STEP 1. $\operatorname{ev}\left(H \times\left(\alpha_{g}-\epsilon\right) \mathbf{1}_{k}\right) \subset G$. Let $b \in Y$, then

$$
\mathrm{ev}\left(H \times\left(\alpha_{g}-\epsilon\right) \mathbf{1}_{k}\right)(b)=\sup _{(f, x): x \in k, f(x)=b} H(f) \wedge\left(\alpha_{g}-\epsilon\right) .
$$

Let $\delta>0$, then there are $f_{\delta}, x_{\delta} \in k$ such that $f_{\delta}\left(x_{\delta}\right)=b$ and

$$
\mathrm{ev}\left(H \times\left(\alpha_{g}-\epsilon\right) \mathbf{1}_{k}\right)(b)-\delta \leq H\left(f_{\delta}\right) \wedge\left(\alpha_{g}-\epsilon\right) .
$$

We choose $k \in\{1,2, \ldots, n\}$ such that $O_{x_{\epsilon}^{k}}\left(x_{\delta}\right) \geq \alpha_{g}-\epsilon$. Then

$$
\begin{aligned}
& \operatorname{ev}\left(H \times\left(\alpha_{g}-\epsilon\right) \mathbf{1}_{k}\right)(b)-\delta \\
\leq & H\left(f_{\delta}\right) \wedge O_{x_{\epsilon}^{k}}\left(x_{\delta}\right) \leq \mathrm{ev}^{-1}(G)\left(f_{\delta}, x_{\delta}\right) \\
& =G\left(f_{\delta}\left(x_{\delta}\right)\right)=G(b) .
\end{aligned}
$$

The arbitrariness of $\delta>0$ now yields the assumption.

STEP 2. For every $x \in k$, we have $G(f(x)) \geq \operatorname{ev}\left(H \times\left(\alpha_{g}-\epsilon\right) \mathbf{1}_{k}\right)(f(x)) \geq H(f) \wedge$ $\left(\alpha_{g}-\epsilon\right)$. Hence, $\left(\mathbf{1}_{k}, G\right)(f) \geq H(f) \wedge\left(\alpha_{g}-\epsilon\right)$. The arbitrariness of $\epsilon>0$ now yields $\left(\mathbf{1}_{k}, G\right) \supset H \cap \alpha_{g}$. As, moreover, $H(g)=\bigcap_{k=1}^{n} H_{\chi_{\epsilon}^{k}}(g) \geq \alpha_{g}-(\epsilon / 2)$, we finally get, again by the arbitrariness of $\epsilon>0, H(g) \wedge \alpha_{g}=\left(\mathbf{1}_{k}, G\right)(g)$. Lemma 1 now yields $\left(\mathbf{1}_{k}, G\right) \in \mathbf{T}$. Hence, $\Delta_{\text {co }} \leq \mathrm{T}$ and the proposition is proved. 


\section{REFERENCES}

[1] I. W. Alderton, Function spaces in fuzzy topology, Fuzzy Sets and Systems 32 (1989), no. 1, 115-124. MR 90i:54013. Zbl 691.54004.

[2] S. Dang and A. Behera, On fuzzy compact-open topology, Fuzzy Sets and Systems 80 (1996), no. 3, 377-381. CMP 96 13. Zbl 868.54003.

[3] G. Jäger, Compactness in fuzzy function spaces, Quaestiones Mathematica, to appear.

[4] Compactness and connectedness as absolute properties in fuzzy topological spaces, Fuzzy Sets and Systems 94 (1997), 405-410.

[5] _ Compactness in fuzzy convergence spaces, Fuzzy Sets and Systems 90 (1998), 341-348. MR 98j\#54006. Zbl 917.54007.

[6] E. Lowen and R. Lowen, Characterization of convergence in fuzzy topological spaces, Internat. J. Math. Math. Sci. 8 (1985), no. 3, 497-511. MR 87b:54006. Zbl 593.54005.

[7] _ A topological universe extension of FTS, Applications of category theory to fuzzy subsets (Dordrecht), Theory Decis. Lib. Ser. B Math. Statist. Methods, vol. 14, Kluwer Acad. Publ., 1992, pp. 153-176. MR 93d:18011. Zbl 774.54002.

[8] E. Lowen, R. Lowen, and P. Wuyts, The categorical topology approach to fuzzy topology and fuzzy convergence, Fuzzy Sets and Systems 40 (1991), no. 2, 347-373. MR 92f:54005. Zbl 728.54001.

[9] R. Lowen, Fuzzy topological spaces and fuzzy compactness, J. Math. Anal. Appl. 56 (1976), no. 3, 621-633. MR 55 13357. Zbl 342.54003.

[10] _ Convergence in fuzzy topological spaces, General Topology Appl. 10 (1979), no. 2, 147-160. MR 80b:54006. Zbl 409.54008.

[11] YuWei Peng, Topological structure of a fuzzy function space-the pointwise convergent topology and compact open topology, Kexue Tongbao (English Ed.) 29 (1984), no. 3, 289-292. MR 86g:54014. Zbl 551.54006.

[12] H. Poppe, Compactness in general function spaces, VEB Deutscher Verlag der Wissenschaften, Berlin, 1974. MR 55 11199. Zbl 291.54012.

[13] GuoJun Wang, A new fuzzy compactness defined by fuzzy nets, J. Math. Anal. Appl. 94 (1983), no. 1, 1-23. MR 85k:54005. Zbl 512.54006.

JÄGER: LESSINGSTRASSE 13, D-76135 KARLSRUHE, GERMANY

Current address: Deutsches Zentrum Für Luft und Raumfahrt DLR, Institut Für Hochfrequenztechnik, Postfach 11 16, D-82230 Wessling, Germany

E-mail address: gjaegar@dv.op.d7r.de 


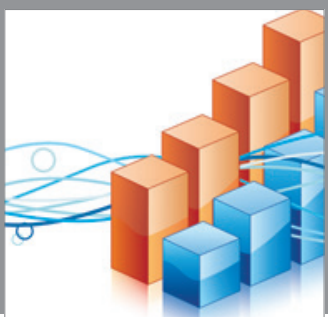

Advances in

Operations Research

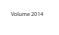

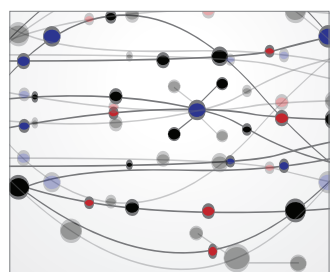

\section{The Scientific} World Journal
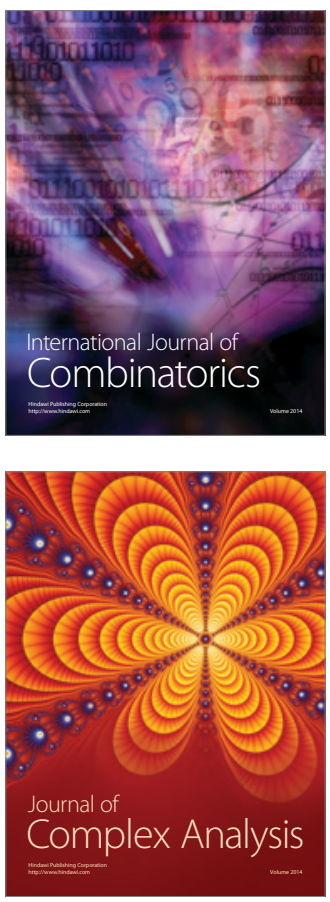

International Journal of

Mathematics and

Mathematical

Sciences
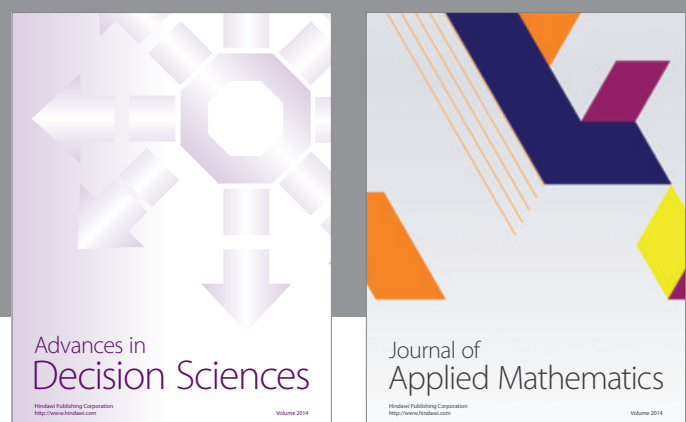

Journal of

Applied Mathematics
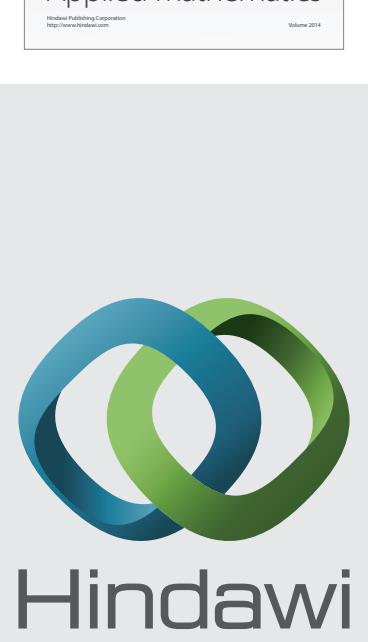

Submit your manuscripts at http://www.hindawi.com
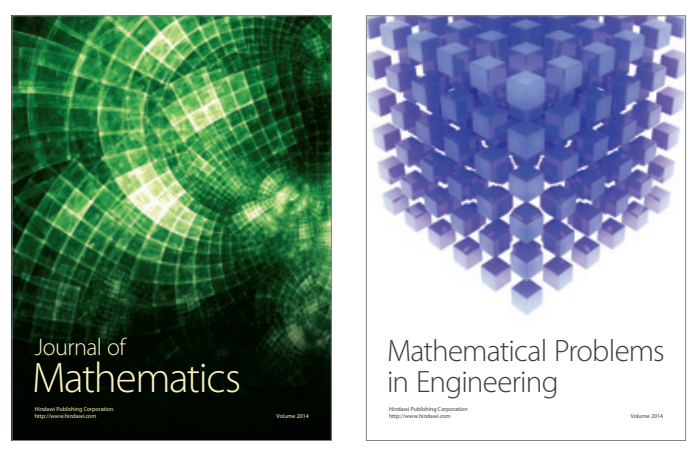

Mathematical Problems in Engineering
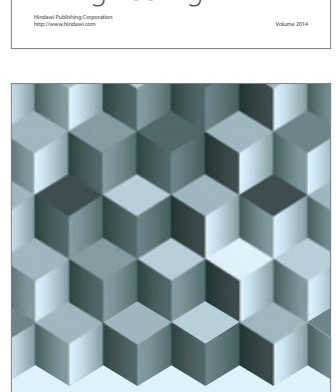

Journal of

Function Spaces
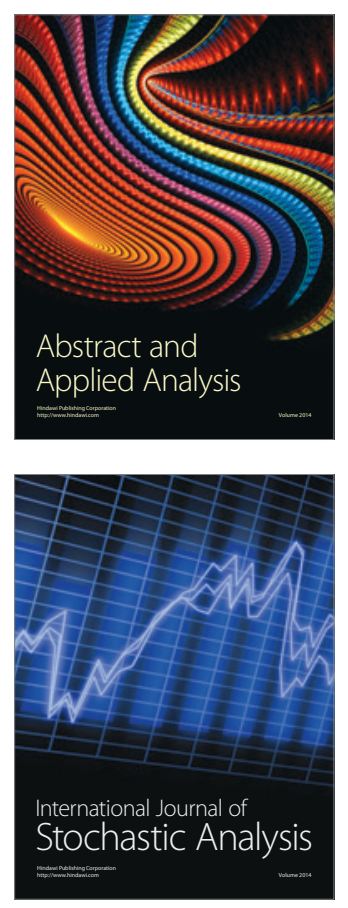

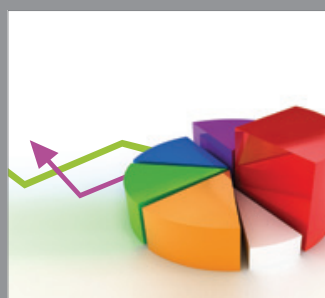

ournal of

Probability and Statistics

Promensencen
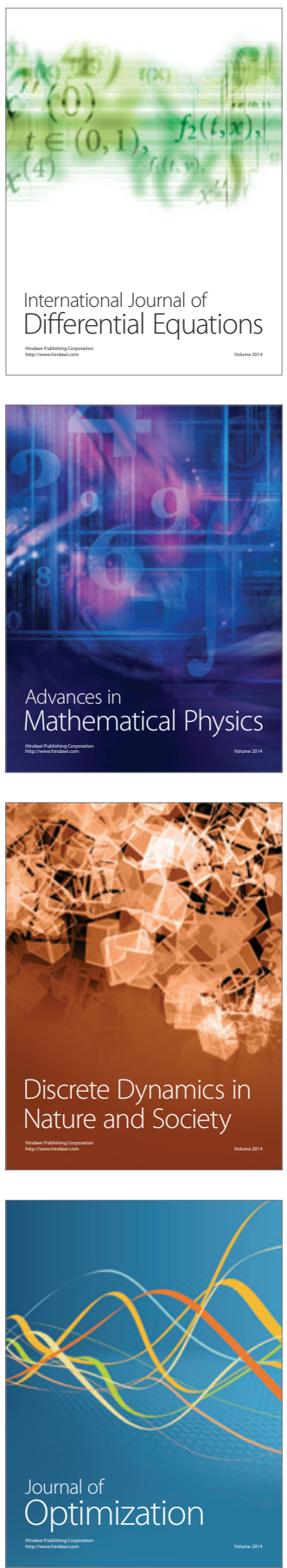